\title{
Species check-list for Tintinnids of the Philippines Archipelago (Protozoa, Ciliophora)
}

\author{
Jane Abigail Santiago', Maria Carmen Lagman' \\ I De La Salle University, Taft Avenue, Manila \\ Corresponding author: Maria Carmen Lagman (ma.carmen.lagman@dlsu.edu.ph)
}

Academic editor: P. Stoev | Received 5 March 2018 | Accepted 17 May 2018 | Published 5 July 2018

http://zoobank.org/4CBF404C-353D-4187-802D-501CA08123F5

Citation: Santiago JA, Lagman MC (2018) Species check-list for Tintinnids of the Philippines Archipelago (Protozoa, Ciliophora). ZooKeys 771: 1-14. https://doi.org/10.3897/zookeys.771.24806

\begin{abstract}
Tintinnids are an essential link between nano- and macro- planktons in the food webs of the marine environment. It is also known that tintinnids are one of the major components of marine planktonic ciliates and has a cosmopolitan character. In the Philippine archipelago, which is recognized as a center of marine biodiversity, tintinnids checklist has not been done or published. Therefore, a checklist is presented in this study based on a compilation of previous tintinnids studies conducted at the Philippines waters. As a result of the studies done since 1941 up to present, a total of 114 taxa belonging to 14 families and 37 genera were listed. The Philippines coastal waters record a total of 50 species while the open seas document 72 species to date.
\end{abstract}

\section{Keywords}

Ciliates, list, Manila Bay, Philippine Sea, plankton, zooplankton

\section{Introduction}

Microzooplankton $(20-200 \mu \mathrm{m})$ constitute a major component of the marine plankton community. Previously, the significance of microzooplankton (MZP) was commonly linked with microbial loop and corresponding microbial web (Calbet and Landry 2004, Calbet et al. 2008), but recent studies have shown that they also play a key 
role in the herbivorous food web (Dolan et al. 2007, Putland and Iverson 2007). MZP graze a wide variety of particles from bacteria to nano- and phytoplankton as well as other similar organisms. They have a crucial role in the first feeding of the larval fishes (Stoecker and Capuzzo 1990, Fukami et al. 1999) and thus should be valued in the aquaculture industry. The awareness of the dynamic role of MZP in marine ecosystem resulted in the increase of scientific interest in the factors affecting their abundance and distribution. Research on microzooplankton arises as one of the vital parts of biological oceanography. In order to fully understand MZP behavior in different environments, a systematic qualitative study that includes listing of the species in a region is an essential step in exploring these organisms.

One of the best-known groups of marine microzooplanktonic ciliates is tintinnid (Kato and Taniguchi 1993). The distinctive characteristic of the tintinnid is its lorica, which has been the basis of their identification and classification. The easiness in identifying tintinnids based on their morphological features made them model specimens for research on species distributions, diversity, and variations in the structure of microzooplankton communities (Dolan and Gallegos 2001). Studies about the tintinnids distribution are essential due to the fact that they have been used as bio-indicators of different water massess (Kim et al. 2012). For example, the tintinnid species named Epiplocyloides reticulata (Ostenfeld \& Schmidt, 1901) has been acknowledged as the Kuroshio water current indicator (Lee and Kim 2010). Records of $E$. reticulata are important to know the geographic extension of the warm Kuroshio current and the possible areas it can affect. A documentation of the tintinnid distribution is recognized as one of the best method to trace the flow of the water mass in open oceans and coastal waters (Lee and Kim 2010). In an archipelagic country such as Philippines, conducting tintinnid studies can be helpful in tracing different water masses and can aid in the assessment and management of its marine environment. However, tintinnids are poorly studied in the Philippines, a place which has been recognized as the center of the center of marine shore fish biodiversity (Carpenter and Springer 2005). A species- checklist for tintinnids specific for the Philippines can be a good starting point for any researcher who wants to conduct a tintinnid survey or any type of investigation in the country.. In order to assist other possible and future tintinnids studies in the Philippines, this present work aims to present the first and current checklist of tintinnid species in the Philippines. The authors also made this list to encourage other researcher to increase tintinnid studies in the Philippines. This study is based on a compilation of the literature to date.

\section{Materials and methods}

The Philippines archipelago is bound by the Bashi Channel to the north, the Philippine Sea to the east and northeast, the Celebes Sea to the south, the Sulu Sea to the southwest, and the South China Sea to the west and northwest side.

In this study, all published literature from 1941 to 2017 was examined. Taxonomical species and author names were written according to Roxas (1941), Gómez (2007), 


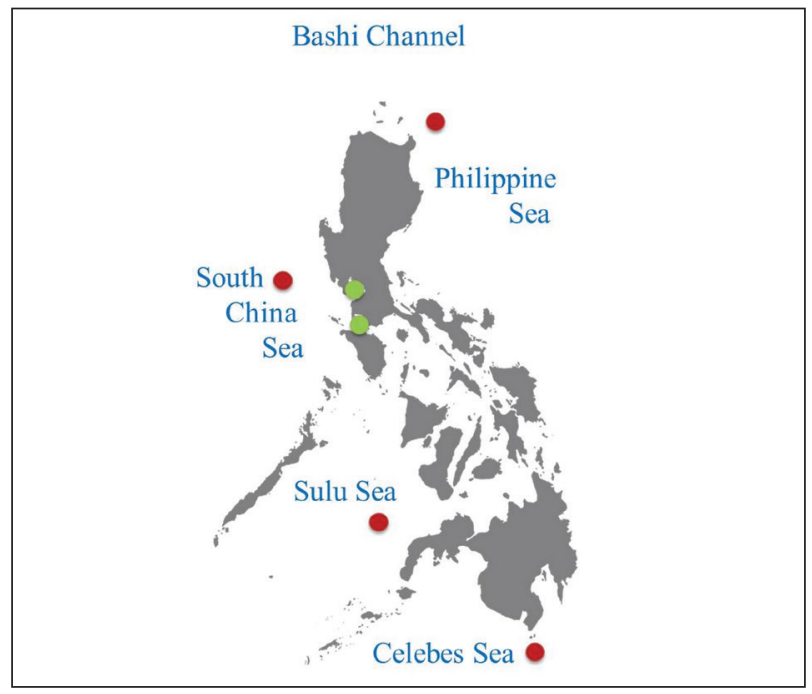

Figure I. Map of the Philippines. Dots imdicate the sites with recorded tintinnid species. Key: green dots: coastal water; red dots: open sea.)

Kim et al. (2012) and Santiago et al. (2017). The study of Taniguchi (1977) was not included as a reference in enumerating tintinnid species since he only referred tintinnids as a group and his paper does not contain any detailed list of tintinnid species. The WoRMS (World Register of Marine Species) data system (Warren 2018) was used for classification and basis of the current species name. The species checklist in this study is alphabetically ordered.

\section{Results}

In related studies conducted in the Philippines, 114 tintinnid species belonging to $14 \mathrm{fam}$ ilies and 37 genera have been recorded. The families Codonellidae (22 species, 19.30\%) and Tintinnidae (21 species, 18.42\%) have the highest recorded species (Table 3). The systematic list and biogeographical distribution of the species are presented below:

\section{Kingdom: Chromista}

Subkingdom: Harosa

Phylum: Ciliophora Doflein, 1901

Class: Oligotrichea Bütschli, 1887

Subclass: Oligotrichia Bütschli, 1887

Order: Choreotrichida Small \& Lynn, 1985

Family: Ascampbelliellidae Corliss, 1960

Genus: Acanthostomella Jörgensen, 1927

Acanthostomella conicoides Kofoid \& Campbell, 1929

Acanthostomella minutissima Kofoid \& Campbell, 1929 
Genus: Ascampbelliella Corliss, 1960

Ascampbelliella acuta (Kofoid \& Campbell, 1929)

Ascampbelliella armilla (Kofoid \& Campbell, 1929)

Ascampbelliella retusa (Hada, 1935)

Ascampbelliella urceolata (Ostenfeld, 1899)

Genus: Craterella Kofoid \& Campbell, 1929

Craterella aperta Marshall

Family: Codonellidae Kent, 1881

Genus: Codonaria Kofoid \& Campbell, 1939

Codonaria oceanica (Brandt, 1906)

Genus: Codonella Haeckel, 1873

Codonella amphorella Biedermann, 1893

Genus: Poroecus Cleve, 1902

Poroecus annulatus Kofoid \& Campbell, 1929

Poroecus apicatus Kofoid \& Campbell, 1929

Genus: Tintinnopsis Stein, 1867

Tintinnopsis bacoornensis Roxas, 1941

Tintinnopsis beroidea Stein, 1867

Tintinnopsis buetschlii Daday, 1887

Tintinnopsis campanula Ehrenberg, 1840

Tintinnopsis chinglanensis Nie \& Cheng, 1947

Tintinnopsis corniger Hada, 1964

Tintinnopsis cylindrica Daday, 1887

Tintinnopsis directa Hada, 1932

Tintinnopsis gracilis Kofoid \& Campbell, 1929

Tintinnopsis loricata Brandt, 1906

Tintinnopsis major Meunier, 1910

Tintinnopsis manilensis Roxas, 1941

Tintinnopsis mortensenii Schmidt, 1902

Tintinnopsis radix (Imhof, 1886)

Tintinnopsis rotundata Kofoid \& Campbell, 1929

Tintinnopsis tocantinensis Kofoid \& Campbell, 1929

Tintinnopsis turgida Kofoid \& Campbell, 1929

Tintinnopsis uruguayensis Balech, 1948

Family: Codonellopsidae Kofoid \& Campbell, 1929

Genus: Codonellopsis Jörgensen, 1924

Codonellopsis morchella (Cleve) Jörgensen, 1924

Codonellopsis orthoceras (Haeckel, 1873) Jörgensen, 1924

Codonellopsis ostenfeldi (Schmidt, 1902) Kofoid \& Campbell, 1929

Codonellopsis pusilla (Cleve) Jörgensen, 1924

Codonellopsis schabi (Brandt, 1906) Kofoid \& Campbell, 1929

Family: Cyttarocylididae Kofoid \& Campbell, 1939

Genus: Cyttarocylis Fol, 1881

Cyttarocylis cassis (Haeckel, 1837) 
Family: Dictyocystidae Haeckel, 1873

Genus: Wangiella Nie, 1934

Wangiella dicollaria $\mathrm{Nie}, 1934$

Genus: Dictyocysta Ehrenberg, 1854

Dictyocysta elegans Ehrenberg, 1854

Dictyocysta mitra Haeckel, 1873

Family: Epiplocylididae Kofoid \& Campbell, 1939

Genus: Epiplocylis Jörgensen, 1924

Epiplocylis calyx (Brandt, 1906)

Epiplocylis exquisita Kofoid \& Campbell, 1929

Epiplocylis undella (Ostenfeld \& Schmidt) Jörgensen, 1927

Genus: Epiplocyloides Hada, 1938

Epiplocyloides acuta (Kofoid \& Campbell,1929)

Epiplocyloides ralumensis (Brandt, 1906)

Epiplocyloides reticulata (Ostenfeld \& Schmidt, 1901)

Family: Metacylididae Kofoid \& Campbell, 1929

Genus: Coxliella Brandt

Coxliella longa Kofoid \& Campbell, 1929

Coxliella mariana Hada, 1938

Genus: Metacylis Jörgensen, 1924

Metacylis hemisphaerica Roxas, 1941

Metacylis jörgensenii (Cleve) Kofoid \& Campbell, 1929

Metacylis kofoidi Roxas, 1941

Metacylis tropica Duran, 1957

Genus: Helicostomella Jörgensen, 1924

Helicostomella longa (Brandt, 1906)

Genus: Climacocylis Jörgensen, 1924

Climacocylis elongata Kofoid \& Campbell, 1929

Climacocylis cf. leospiralis Kofoid \& Campbell

Climacocylis scalaria Brandt, 1906

Climacocylis sipho (Brandt, 1906) Kofoid \& Campbell, 1929

Family: Petalotrichidae Kofoid \& Campbell, 1929

Genus: Petalotricha Kent, 1881

Petalotricha major Jörgensen, 1925

Family: Ptychocylididae Kofoid \& Campbell, 1929

Genus: Favella Jörgensen, 1924

Favella ehrenbergii (Claparède \& Lachmann, 1858) Jörgensen, 1924

Favella simplex Roxas, 1941

Favella philippinensis Roxas, 1941

Favella elongata Roxas, 1941

Favella azorica (Cleve, 1900) Jörgensen, 1924

Family: Rhabdonellidae Kofoid \& Campbell, 1929

Genus: Rhabdonella Brandt, 1906

Rhabdonella amor (Cleve, 1900) Brandt, 1907 
Rhabdonella apophysata Jörgensen, 1924

Rhabdonella brandti Kofoid \& Campbell, 1929

Rhabdonella conica Kofoid \& Campbell, 1929

Rhabdonella cornucopia Kofoid \& Campbell, 1929

Rhabdonella elegans Jörgensen, 1924

Rhabdonella exilis Kofoid \& Campbell, 1929

Rhabdonella sanyahensis Nie \& Cheng, 1947

Rhabdonella fenestrata Roxas, 1941

Rhabdonella valdestriata (Brandt) Kofoid \& Campbell, 1929

Rhabdonella spiralis (Fol, 1881)

Genus: Protorhabdonella Jörgensen, 1924

Protorhabdonella curta Cleve, 1900

Protorhabdonella simplex (Cleve) Jörgensen, 1924

Protorhabdonella striatura Kofoid \& Campbell, 1929

Family: Tintinnidae Claparède \& Lachmann, 1858

Genus: Amphorellopsis Kofoid \& Campbell, 1929

Amphorellopsis acuta (Schmidt, 1902)

Genus: Amphorides Strand, 1928

Amphorides amphora (Claparède \& Lachmann, 1858)

Amphorides quadrilineata (Claparède \& Lachmann, 1858)

Amphorides minor Jörgensen, 1924

Genus: Brandtiella Kofoid \& Campbell, 1929

Brandtiella palliata (Brandt, 1906) Kofoid \& Campbell, 1929

Genus: Canthariella (Kofoid \& Campbell, 1929)

Canthariella pyramidata (Jörgensen, 1924) Kofoid \& Campbell, 1929

Genus: Dadayiella Kofoid \& Campbell, 1929

Dadayiella ganymedes (Entz, 1884) Kofoid \& Campbell, 1929

Dadayiella pachytoecus (Dendy, 1924)

Genus: Eutintinnus Kofoid \& Campbell, 1939

Eutintinnus apertus Kofoid \& Campbell, 1929

Eutintinnus fraknoii (Daday, 1887)

Eutintinnus lusus-undae (Entz, 1885)

Eutintinnus stramentus (Kofoid \& Campbell, 1929)

Genus Ormosella Kofoid \& Campbell, 1929

Ormosella haeckeli Kofoid \& Campbell, 1929

Genus: Salpingella Jörgensen, 1924

Salpingella acuminata (Claparède \& Lachmann, 1858) Jörgensen, 1924

Salpingella acuminatoides (Laackmann) Kofoid \& Campbell, 1929

Salpingella attenuata Kofoid \& Campbell, 1929

Salpingella decurtata Jörgensen, 1924

Salpingella subconica Kofoid \& Campbell, 1929 
Genus: Steenstrupiella Kofoid \& Campbell, 1929

Steenstrupiella intumescens (Jörgensen, 1924) Kofoid \& Campbell, 1929

Steenstrupiella steenstrupii (Claparède \& Lachmann, 1858) Kofoid \& Campbell, 1929

Genus: Tintinnus Schrank, 1803

Tintinnus perminutus Kofoid \& Campbell, 1929

Family: Tintinnidiidae Kofoid \& Campbell

Genus: Tintinnidium Kent, 1881

Tintinnidium primitivum Busch, 1923

Tintinnidium cylindrica Daday, 1886

Tintinnidium ampullarium Roxas, 1941

Genus: Leprotintinnus Jörgensen, 1899

Leprotintinnus nordqvistii (Brandt, 1906) Kofoid \& Campbell, 1929

Leprotintinnus tubulosus Roxas, 1941

Family: Undellidae Kofoid \& Campbell, 1929

Genus: Undella Daday, 1887

Undella claparedei (Entz) Daday, 1887

Undella clevei Jörgensen, 1924

Undella hyalina Daday, 1887

Undella subcaudata Jörgensen, 1924

Family: Xystonellidae Kofoid \& Campbell, 1929

Genus: Parundella Jörgensen, 1924

Parundella aculeata (Joergensen, 1924)

Parundella caudata (Ostenfeld, 1899) Jörgensen, 1924

Parundella inflata Kofoid \& Campbell, 1929

Parundella longa Joergensen, 1924

Genus: Xystonella Brandt, 1907

Xystonella treforti (Daday, 1887)

Genus: Xystonellopsis Jörgensen, 1924

Xystonellopsis brandti (Laackmann) Jörgensen, 1924

Xystonellopsis cymatica (Brandt, 1906) Jörgensen, 1924

Xystonellopsis dahli (Brandt, 1906) Kofoid \& Campbell, 1929

Xystonellopsis paradoxa (Cleve, 1900) Jörgensen, 1924

The study of Roxas (1941) contained the first recorded tintinnid species in the Philippines. Roxas (1941) documented 32 tintinnid species wherein ten were newly discovered species (Table 2). Favella simplex, Favella philippinensis, and Favella elongata were the only accepted and registered species in the WoRMS database (Warren 2018) among the said newly discovered species. The other newly discovered species are still included in this present checklist due to the scarcity of tintinnid studies in the Philippines. The other newly discovered species were not recorded in any other studies and 
we took into consideration that they might be endemic in the area where Roxas (1941) collected them. Roxas also misspelled Leprotinntinnus nordqvistii, which he recorded as Leprotinntinnus nordquisti.

Since 1941, only three other studies (Gómez 2007, Kim et al. 2012, Santiago et al. 2017) were made in the Philippines that identified tintinnids to species level. The paper of Roxas (1941) and Santiago et al. (2017) recorded a total of 50 tintinnid species from coastal waters of Manila Bay (39 species) and Puerto Galera Bay (11 species). On the other hand, Gómez (2007) and Kim et al. (2012) conducted their sample collection within the Philippines open seas, which amounted to 72 tintinnid species.

Tintinnopsis, Codonellopsis, Coxliella, Metacylis, Rhabdonella, Epiplocylis and Eutintinus were the genera that both appeared in coastal and open waters (Table 1). There were eight genera that were only recorded in coastal waters and a total of 24 genera were solely found in the open seas (Table 1). Epiplocylis undella and Rhabdonella spiralis were the only species common to all of four tintinnid studies in the Philippines (Table 2).

\section{Discussion}

Presently, there are only four related studies (Roxas 1941, Gómez 2007, Kim et al. 2012, Santiago et al. 2017) that contain tintinnid species in the Philippines. Roxas (1941) and Santiago et al. (2017) conducted their zooplankton collection within the Philippines coastal waters while Gómez (2007) and Kim et al. (2012) had cruises along the open seas. Table 1 and 2 showed the tintinnids distribution between open seas and coastal waters. This is an important data because some of the tintinnids were categorized into biogeographical groups (Pierce and Turner 1993). The studies (Lee and Kim 2010, Kim et al. 2012) that utilized tintinnids as indicator species used their biogeographical groups to assess water quality and mass movements. In this present study, there are species and genera that were only recorded in one area and some both appeared in open seas and coastal waters. Hence, the variation of the tintinnids distribution between open seas and coastal waters in this current work might help in further classification of tintinnid species to their biogeographical groups.

It should also be noted that each of the said four studies had a different sampling technique and effort. Roxas (I94I) towed a no. 20 plankton net with 176 mesh per inch which means that it has an aperture of $0.076 \mathrm{~mm}$ or $76 \mu \mathrm{m}$. The plankton net that Santiago et al. 2017 used has $64 \mu \mathrm{m}$ mesh size. These can indicate that the majority of the collected species of Roxas (1941) and Santiago et al. 2017 were large tintinnid species $(>64 \mu \mathrm{m})$. Microzooplankton size range from 20 to $200 \mu \mathrm{m}$, thus, collecting tintinnids through plankton net with a relatively larger aperture size can result in loss of most of the smaller-sized tintinnids.

In the studies conducted in Philippines open seas, Gómez (2007) used Niskin bottles while Kim et al. (2012) towed a $20 \mu \mathrm{m}$ mesh-plankton-net. The differences in methodologies and lack of standardization of sampling technique on tintinnids collection (Gómez 2007) can add complication on the analysis and comparison of 
Table I. Summary of the tintinnid appearance between coastal and open seas by genus.

\begin{tabular}{|c|c|c|c|}
\hline & Coastal & Open sea & Both \\
\hline & Favella & Acanthostomella & Codonellopsis \\
\hline & Helicostomella & Amphorellopsis & Coxliella \\
\hline & Leprotintinnus & Amphorides & Epiplocylis \\
\hline & Petalotricha & Ascampbelliella & Eutintinnus \\
\hline & Tintinnidium & Brandtiella & Metacylis \\
\hline & Tintinnus & Canthariella & Rhabdonella \\
\hline & Wangiella & Climacocylis & Tintinnopsis \\
\hline & & Codonaria & \\
\hline & & Codonella & \\
\hline & & Craterella & \\
\hline & & Cyttarocylis & \\
\hline & & Dadayiella & \\
\hline & & Dictyocysta & \\
\hline & & Epiplocylididae & \\
\hline & & Epiplocyloides & \\
\hline & & Ormosella & \\
\hline & & Parundella & \\
\hline & & Poroecus & \\
\hline & & Protorhabdonella & \\
\hline & & Salpingella & \\
\hline & & Steenstrupiella & \\
\hline & & Undella & \\
\hline & & Xystonella & \\
\hline & & Xystonellopsis & \\
\hline Total & 7 & 24 & 7 \\
\hline
\end{tabular}

Table 2. Distribution of tintinnid species reported in the Philippines. The open sea has records from the southwest (SW) seas that include Sulu, Celebes and South China Sea (Gómez 2007). The northeast (NE) was based on the study of Kim et al. (2012) in the Philippine Sea. The species in the Coastal areas were from Manila bay (MB) (Roxas 1941, Santiago et al. 2017) and Puerto Galera Bay (PG) (Roxas 1941). An asterisk $\left({ }^{*}\right)$ denotes new species.

\begin{tabular}{l|c|c|c|c}
\hline \multirow{2}{*}{ Taxon } & \multicolumn{2}{|c|}{ Open sea } & \multicolumn{2}{c}{ Coastal } \\
\cline { 2 - 5 } & SW & NE & MB & PG \\
\hline 1. Acanthostomella conicoides & & + & & \\
\hline 2.Acanthostomella minutissima & + & & & \\
\hline 3. Amphorellopsis acuta & & + & & \\
\hline 4. Amphorides amphora & + & + & & \\
\hline 5. Amphorides minor & & + & & \\
\hline 6. Amphorides quadrilineata & + & + & & \\
\hline 7. Ascampbelliella acuta & & + & & \\
\hline 8. Ascampbelliella armilla & + & & & \\
\hline 9. Ascampbelliella retusa & + & & & \\
\hline 10. Ascampbelliella urceolata & & + & & \\
\hline
\end{tabular}




\section{Taxon}

11. Brandtiella palliata

12. Canthariella pyramidata

13. Climacocylis of. leospiralis

14. Climacocylis elongata

15. Climacocylis scalaria

16. Climacocylis sipho

17. Codonaria oceanica

18. Codonella amphorella

19. Codonellopsis morchella

20. Codonellopsis orthoceras

21. Codonellopsis ostenfeldi

22. Codonellopsis pusilla

23. Codonellopsis schabi

24. Coxliella longa

25. Coxliella mariana

26. Craterella aperta

27. Cyttarocylis cassis

28. Dadayiella ganymedes

29. Dadayiella pachytoecus

30. Dictyocysta elegans

31. Dictyocysta mitra

32. Epiplocylis calyx

33. Epiplocylis exquisita

34. Epiplocylis undella

35. Epiplocyloides acuta

36. Epiplocyloides ralumensis

37. Epiplocyloides reticulata

38. Eutintinnus apertus

39. Eutintinnus fraknoii

40. Eutintinnus lusus-undae

41. Eutintinnus stramentus

42. Favella azorica

43. Favella ehrenbergii

44. Favella elongate*

45. Favella philippinensis*

46. Favella simplex*

47. Helicostomella longa

48. Leprotintinnus nordqvistii

49. Leprotintinnus tubulosus*

50. Metacylis hemisphaerica*

51. Metacylis jörgensenii

52. Metacylis kofoidi*

53. Metacylis tropica

54. Ormosella haeckeli

55. Parundella aculeata

56. Parundella caudata

\begin{tabular}{|c|c|c|c|}
\hline \multicolumn{2}{|c|}{ Open sea } & \multicolumn{2}{|c|}{ Coastal } \\
\hline SW & $\mathrm{NE}$ & MB & PG \\
\hline+ & + & & \\
\hline+ & + & & \\
\hline \multicolumn{4}{|l|}{+} \\
\hline & + & & \\
\hline \multirow[t]{2}{*}{+} & + & & \\
\hline & + & & \\
\hline \multicolumn{4}{|l|}{+} \\
\hline & + & & \\
\hline & & + & \\
\hline & + & + & \\
\hline & & + & \\
\hline \multicolumn{4}{|l|}{+} \\
\hline \multicolumn{4}{|l|}{+} \\
\hline & & + & \\
\hline & + & & \\
\hline \multicolumn{4}{|l|}{+} \\
\hline & + & & \\
\hline \multirow[t]{2}{*}{+} & + & & \\
\hline & + & & \\
\hline \multirow[t]{4}{*}{+} & + & & \\
\hline & + & & \\
\hline & + & & \\
\hline & & & + \\
\hline \multirow[t]{3}{*}{+} & + & + & + \\
\hline & + & & \\
\hline & & & + \\
\hline \multicolumn{4}{|l|}{+} \\
\hline \multicolumn{4}{|l|}{+} \\
\hline+ & + & + & \\
\hline+ & + & + & \\
\hline \multirow[t]{14}{*}{+} & + & & \\
\hline & & & + \\
\hline & & + & \\
\hline & & + & \\
\hline & & + & \\
\hline & & + & \\
\hline & & + & \\
\hline & & + & \\
\hline & & + & \\
\hline & & & + \\
\hline & + & + & \\
\hline & & & + \\
\hline & & + & \\
\hline & + & & \\
\hline \multicolumn{4}{|l|}{+} \\
\hline & + & & \\
\hline
\end{tabular}




\begin{tabular}{|c|c|c|c|c|}
\hline \multirow{2}{*}{ Taxon } & \multicolumn{2}{|c|}{ Open sea } & \multicolumn{2}{|c|}{ Coastal } \\
\hline & SW & NE & MB & PG \\
\hline 57. Parundella inflata & & + & & \\
\hline 58. Parundella longa & + & & & \\
\hline 59. Petalotricha major & & & & + \\
\hline 60. Poroecus annulatus & + & & & \\
\hline 61. Poroecus apicatus & & + & & \\
\hline 62. Protorhabdonella curta & + & + & & \\
\hline 63. Protorhabdonella simplex & + & + & & \\
\hline 64. Protorhabdonella striatura & & + & & \\
\hline 65. Rhabdonella amor & + & + & & + \\
\hline 66. Rhabdonella apophysata & + & + & & \\
\hline 67. Rhabdonella brandti & & & & + \\
\hline 68. Rhabdonella conica & & & + & \\
\hline 69. Rhabdonella cornucopia & & + & & \\
\hline 70. Rhabdonella elegans & + & & & \\
\hline 71. Rhabdonella exilis & & + & & \\
\hline 72. Rhabdonella fenestrata* & & & & + \\
\hline \multicolumn{5}{|l|}{ 73. Rhabdonella sanyahensis } \\
\hline 74. Rhabdonella spiralis & + & + & + & + \\
\hline 75. Rhabdonella valdestriata & & + & & \\
\hline 76. Salpingella acuminata & + & + & & \\
\hline 77. Salpingella acuminatoides & & + & & \\
\hline 78. Salpingella attenuata & + & & & \\
\hline 79. Salpingella decurtata & + & & & \\
\hline 80. Salpingella subconica & & + & & \\
\hline 81. Steenstrupiella intumescens & & + & & \\
\hline 82. Steenstrupiella steenstrupii & + & + & & \\
\hline 83. Tintinnidium ampullarium ${ }^{*}$ & & & + & \\
\hline 84. Tintinnidium cylindrica & & & + & \\
\hline 85. Tintinnidium primitivum & & & + & \\
\hline 86. Tintinnopsis bacoornensis* & & & + & \\
\hline 87. Tintinnopsis beroidea & & & + & \\
\hline 88. Tintinnopsis buetschlii & & & + & \\
\hline 89. Tintinnopsis campanula & + & & & \\
\hline 90. Tintinnopsis chinglanensis & & & + & \\
\hline 91. Tintinnopsis corniger & & & + & \\
\hline 92. Tintinnopsis cylindrica & & & + & \\
\hline 93. Tintinnopsis directa & & & + & \\
\hline 94. Tintinnopsis gracilis & & & + & \\
\hline 95. Tintinnopsis loricata & & & + & \\
\hline 96. Tintinnopsis major & & & + & \\
\hline 97. Tintinnopsis manilensis* & & & + & \\
\hline 98. Tintinnopsis mortensenii & & & + & \\
\hline 99. Tintinnopsis radix & & & + & \\
\hline 100. Tintinnopsis rotundata & & & + & \\
\hline 101. Tintinnopsis tocantinensis & & & + & \\
\hline 102. Tintinnopsis turgida & & & + & \\
\hline
\end{tabular}




\begin{tabular}{|c|c|c|c|c|}
\hline \multirow{2}{*}{ Taxon } & \multicolumn{2}{|c|}{ Open sea } & \multicolumn{2}{|c|}{ Coastal } \\
\hline & SW & $\mathrm{NE}$ & MB & PG \\
\hline 103. Tintinnopsis uruguayensis & & & + & \\
\hline 104. Tintinnus perminutus & & & + & \\
\hline 105. Undella claparedei & + & + & + & \\
\hline 106. Undella clevei & + & & & \\
\hline 107. Undella hyalina & & + & & \\
\hline 108. Undella subcaudata & + & & & \\
\hline 109. Wangiella dicollaria & & & + & \\
\hline 110. Xystonella treforti & + & + & & \\
\hline 111. Xystonellopsis brandti & & + & & \\
\hline 112. Xystonellopsis cymatica & + & + & & \\
\hline 113. Xystonellopsis dabli & & + & & \\
\hline 114. Xystonellopsis paradoxa & & + & & \\
\hline 108. Undella subcaudata & + & & & \\
\hline 109. Wangiella dicollaria & & & + & \\
\hline 110. Xystonella treforti & + & + & & \\
\hline 111. Xystonellopsis brandti & & + & & \\
\hline 112. Xystonellopsis cymatica & + & + & & \\
\hline 113. Xystonellopsis dahli & & + & & \\
\hline 114. Xystonellopsis paradoxa & & + & & \\
\hline 108. Undella subcaudata & + & & & \\
\hline 109. Wangiella dicollaria & & & + & \\
\hline 110. Xystonella treforti & + & + & & \\
\hline 111. Xystonellopsis brandti & & + & & \\
\hline 112. Xystonellopsis cymatica & + & + & & \\
\hline 113. Xystonellopsis dabli & & + & & \\
\hline 114. Xystonellopsis paradoxa & & + & & \\
\hline
\end{tabular}

Table 3. Percentage (\%) Distribution of Tintinnids families from the Philippines.

\begin{tabular}{l|c|c|c}
\hline Family & Genus & Species & \% \\
\hline Ascampbelliellidae & 3 & 7 & 6.14 \\
\hline Codonellidae & $\mathbf{4}$ & $\mathbf{2 2}$ & $\mathbf{1 9 . 3 0}$ \\
\hline Codonellopsidae & 1 & 5 & 4.39 \\
\hline Cyttarocylididae & 1 & 1 & 0.88 \\
\hline Dictyocystidae & 2 & 3 & 2.63 \\
\hline Epiplocylididae & 2 & 6 & 5.26 \\
\hline Metacylididae & 4 & 11 & 9.65 \\
\hline Petalotrichidae & 1 & 1 & 0.88 \\
\hline Ptychocylididae & 1 & 5 & 4.39 \\
\hline Rhabdonellidae & 2 & 14 & 12.28 \\
\hline Tintinnidae & $\mathbf{1 0}$ & $\mathbf{2 1}$ & $\mathbf{1 8 . 4 2}$ \\
\hline Tintinnidiidae & 2 & 5 & 4.39 \\
\hline Undellidae & 1 & 4 & 3.51 \\
\hline Xystonellidae & 3 & 9 & 7.89 \\
\hline
\end{tabular}


their biogeographical distribution. Apparently, more studies on tintinnids in the Philippines and a standard of methodology should be established. The authors executed this current work to serve as a starting point for other researchers and encourage them to conduct studies on tintinnids in a center of marine biodiversity such as the Philippines.

\section{References}

Calbet A, Landry MR (2004) Phytoplankton growth, microzooplankton grazing, and carbon cycling in marine systems. Limnology and Oceanography 49: 51-57. https://doi. org/10.4319/lo.2004.49.1.0051

Calbet A, Trepa I, Almeda R, Salo V, Saiz E, Movilla J, Alcaraz L, Yebra L, Simo R (2008) Impact of micro-and nanograzers on phytoplankton assessed by standard and size-fractionated dilution grazing experiments. Aquatic Microbial Ecology 40: 145-156. https://doi. org/10.3354/ame01171

Dolan JR, Gallegos CL (2001) Estuarine diversity of tintinnids (planktonic ciliates). Journal of Plankton Research 23: 1009-1027. https://doi.org/10.1093/plankt/23.9.1009

Dolan JR, Ritchie ME, Ras J (2007) The "neutral" community structure of planktonic herbivores, tintinnid ciliates of the microzooplankton, across the SE Tropical Pacific Ocean. Biogeosciences Discuss 4: 561-593. https://doi.org/10.5194/bg-4-297-2007

Gómez F (2007) Trends on the distribution of ciliates in the open Pacific Ocean. Acta Oelogica 32: 188-202. https://doi.org/10.1016/j.actao.2007.04.002

Kato S, Taniguchi A (1993) Tintinnid ciliates as indicator species of different water masses in the western North Pacific Polar Front. Fisheries Oceanography 2: 166-174. https://doi. org/10.1111/j.1365-2419.1993.tb00132.x

Carpenter KE, Springer VG (2005) The center of the center of marine shore fish biodiversity: the Philippine Islands. Environmental Biology of Fishes 72: 467-480. https://doi. org/10.1007/s10641-004-3154-4

Fukami KA, Watanabe A, Fujita S, Yamaoka K, Nishijima T (1999) Predation on naked protozoan microzooplankton by fish larvae. Marine Ecology Progress Series 185: 285-291. https://doi.org/10.3354/meps18528

Kim YO, Shin K, Jang PG, Choi HW, Noh JH, Yang EJ, Kim E, Jeon D (2012) Tintinnid species as biological indicators for monitoring intrusion of the warm oceanic waters into Korean coastal waters. Ocean Science Journal 47: 161-172. https://doi.org/10.1007/ s12601-012-0016-4

Lee JB, Kim YH (2010) Distribution of Tintinnids (Loricate Ciliates) in East Asian Waters in Summer. In: Ishimatsu A,Lie H-J (Eds) Coastal Environmental and Ecosystem Issues of the East China Sea. 173-180.

Pierce RW, Turner JT (1993) Global biogeography of marine tintinnids. Marine Ecology Progress Series 94: 11-26. https://doi.org/10.3354/meps094011 
Putland JN, Iverson RL (2007) Microzooplankton: major herbivores in an estuarine planktonic food web. Marine Ecology Progress Series 345: 63-73. https://doi.org/10.3354/ meps06841

Roxas HA (1941) Marine protozoa of the Philippines. Philippine Journal of Science 74: 91-139. Santiago JA, Furio EF, Borja VM, Gatdula NC, Santos MD (2017) First records of tintinnid (Protozoa: Ciliophora: Tintinnina) species in Manila Bay. DLSU Research Congress 2017: The ASEAN Ecosystem @50: Change for a more inclusive growth, June 20-22, 2017, Manila, Philippines CENSER-II-032.

Stoecker DK, Capuzzo JM (1990) Predation on protozoa: its importance to zooplankton. Journal of Plankton Research 12:891-908. https://doi.org/10.1093/plankt/12.5.891

Taniguchi A (1977) Distribution of microzooplankton in the Philippine Sea and the Celebes Sea in summer, 1972. Journal of the Oceanographical Society of Japan 33: 82-89. https:// doi.org/10.1007/BF02110013

Warren A (2018) World Ciliophora Database: WoRMS (World Register of Marine Species). http://www.marinespecies.org/aphia.php?p=taxdetails\&id=415082 [on 2018-02-21] 